\title{
Attendance and Substance Use Outcomes for the Seeking Safety Program: Sometimes Less Is More
}

\author{
Denise A. Hien, \\ Department of Clinical Psychology, Graduate Center, City College of the City University of New \\ York, and Department of Psychiatry, Columbia University College of Physicians and Surgeons
}

\section{Antonio A. Morgan-Lopez,}

L. L. Thurstone Psychometric Laboratory and Department of Psychology, University of North Carolina at Chapel Hill

Aimee N. C. Campbell,

Substance Abuse Division, New York State Psychiatric Institute, New York, New York, and Department of Psychiatry, Columbia University College of Physicians and Surgeons

Lissette M. Saavedra,

Division of Social Policy, Health, and Economics Research, RTI International, Research Triangle Park, North Carolina

\section{Elwin $\mathrm{Wu}$,}

School of Social Work, Columbia University College of Physicians and Surgeons

\section{Lisa Cohen,}

Substance Abuse Division, New York State Psychiatric Institute, New York, New York, and Center for Trauma and Addictions, Department of Psychology, City College of the City University of New York

Lesia Ruglass, and

Center for Trauma and Addictions, Department of Psychology, City College of the City University of New York

\section{Edward V. Nunes}

Substance Abuse Division, New York Sate Psychiatric Institute, New York, New York, and Department of Psychiatry, Columbia University College of Physicians and Surgeons

\section{Abstract}

\begin{abstract}
Objective-This study uses data from the largest effectiveness trial to date on treatment of cooccurring posttraumatic stress and substance use disorders, using advances in statistical methodology for modeling treatment attendance and membership turnover in rolling groups.

Method-Women receiving outpatient substance abuse treatment $(N=353)$ were randomized to 12 sessions of Seeking Safety or a health education control condition. Assessments were completed at baseline and at 1 week, 3, 6, and 12 months posttreatment. Outcome measures were alcohol and cocaine use in the prior 30 days captured using the Addiction Severity Index. Latent class pattern mixture modeling (LCPMM) was used to estimate attendance patterns and to test for treatment effects within and across latent attendance patterns and group membership turnover.
\end{abstract}

(C) 2011 American Psychological Association

Correspondence concerning this article should be addressed to Denise A. Hien, 160 Convent Avenue, North Academic Center 8-131, City College of the City University of New York, New York, NY 10030. dah26@ columbia.edu.

All other authors report no competing interests. 
Results-Across LCPMM analyses for alcohol and cocaine use, similar treatment attendance patterns emerged: Completers never decreased below an $80 \%$ probability of attendance, droppers never exceeded a $41 \%$ probability of attendance, and titrators demonstrated a $50 \%$ to $80 \%$ probability of attendance. Among completers, there were significant decreases in alcohol use from baseline to 1-week posttreatment, followed by nonsignificant increases in alcohol during followup. No differences between treatment conditions were detected. Titrators in Seeking Safety had lower rates of alcohol use from 1-week through 12-month follow-up compared with control participants. Droppers had nonsignificant increases in alcohol during both study phases. Cocaine use findings were similar but did not reach significance levels.

Conclusions-The impact of client self-modulation of treatment dosage and group membership composition may influence behavioral treatment outcomes among this population.

\section{Keywords}

attendance patterns; rolling group; latent class pattern mixture models; posttraumatic stress disorder; substance use disorders

Epidemiological and clinical studies have established that the majority of women in substance abuse treatment have been exposed to interpersonal trauma in their lifetimes (Dansky, Saladin, Brady, Kilpatrick, \& Resnick, 1995; Lincoln, Liebschutz, Chernoff, Nguyen, \& Amaro, 2006; Mills, Lynskey, Teesson, Ross, \& Dark, 2005) and commonly meet diagnosis for co-occurring post-traumatic stress disorder (Brady, 2001; Najavits, Weiss, \& Shaw, 1997). As the extent of such comorbidity and its implications for treatment have come to light, a number of manualized cognitive behavior therapy approaches have been developed to address the complex needs of this population with the aim of improving treatment outcomes.

Empirical support for these cognitive behavioral approaches, such as the Seeking Safety program (Najavits, Weiss, Shaw, \& Muenz, 1998) and substance dependence posttraumatic stress disorder (PTSD) therapy (Triffleman, 2000), has been promising overall. However, the lack of well-controlled and sufficiently powered trials combined with small effect sizes among trials that were controlled (e.g., Hien, Cohen, Miele, Litt, \& Capstick, 2004) have resulted in limited evidence that trauma treatments can significantly impact substance use outcomes. To date, no single treatment study has shown a magnitude of symptom improvement such that it could be recommended among guidelines for best clinical practices among women with co-occurring PTSD and addiction.

As we have detailed elsewhere (Hien, Cohen, \& Campbell, 2009), applying conventional statistical methodologies for randomized controlled trials using an intent-to-treat approach can be problematic when examining the efficacy of treatments for co-occurring disorders because of a number of assumptions typically made in group therapy treatment trials for substance abuse (Feaster, Newman, \& Rice, 2003). These assumptions and the ways that they fail to fit the comorbid PTSD/SUD population are examined and include (a) homogeneity of patient population and treatment response, (b) a dose-response relationship between treatment attendance and outcome, and (c) similarity between patient attendance in groups.

\section{Assumption 1: One Size Fits All}

The first assumption is that patients are homogeneous and will have a common treatment response-that one size fits all (Swift, Callahan, \& Levine, 2009). Yet, clinicians and therapists recognize that women who present for treatment in clinical addictions settings are, by definition, a heterogeneous group characterized by variation in psychological, 
psychiatric, and social impairments (Greenfield et al., 2007; Stewart, Ouimette, \& Brown, 2002). Most randomized trials, however, are designed to examine whether the effects of an experimental group psychotherapy has a superior effect on a particular set of specified substance use outcomes relative to a comparison condition, using an intent-to-treat approach (Feaster et al., 2003). Such approaches typically average across all participants assigned to a treatment arm and ignore the ways that receipt of the treatments themselves may differentially affect outcomes.

\section{Assumption 2: More Treatment Attendance Leads to Better Outcomes}

The second assumption concerns prevailing clinical and research wisdom in general psychopathology treatment suggesting a positive dose-response relationship between the number of sessions attended and salutary treatment response (e.g., 16 -18; Lambert, Hansen, $\&$ Finch, 2001). Given the presenting problems of women with co-occurring PTSD/SUD, however, there are substantial barriers to treatment entry, regular attendance, and successful outcomes (Greenfield et al., 2007; Stewart et al., 2002). On average, clinical trials among women with co-occurring PTSD/SUD retain participants for about half of all scheduled sessions (Brady, 2001; Hien et al., 2004; Hien, Wells, et al., 2009). Clinical experience based on previous treatment trials with this population suggests that some traumatized women, with emotional dysregulation as a core underlying syndrome of PTSD (e.g., Roth, Newman, Pelcovitz, van der Kolk, \& Mandel, 1997), may attend sessions intermittently (either not attending sequentially or attending over a longer period of time) to titrate the emotional distress that can be evoked when working with trauma-focused material. Despite missing consecutive sessions, these women often remain engaged in the treatment process and stay in contact with their providers and/or study staff. This is particularly true if intermittent attendance does not interfere with, or even facilitates, positive treatment outcome among women with this type of comorbidity (e.g., Hien, Wells, et al., 2009). Indeed, although it remains an open question among women with co-occurring PTSD and SUD, there is some evidence that number of sessions attended benefits patients up to a diminishing return in some contexts (Barkham et al., 2006; Feaster et al., 2003).

\section{Assumption 3: Group Membership Attendance Patterns Can Be Considered Independently From Individual Outcomes}

A third assumption involves the independent consideration of group context and individual outcomes when analyzing treatment group data. The most common modality of psychotherapy in addictions treatment settings is group therapy with rolling admission (Morgan-Lopez \& Fals-Stewart, 2008a). Yet, most empirical studies have used individual psychotherapy or closed groups for testing treatment efficacy (Weiss, Jaffe, de Menil, \& Cogley, 2004). By design, participation in group therapy creates a degree of interdependence among the members; indeed, this is one of the defining and desirable qualities of therapy groups. Nonindependence among patients in therapy groups makes analysis and interpretation more complex than methods used to analyze data gathered from individually based treatments (Bryk \& Raudenbush, 1992); this is especially true for open enrollment groups, where membership may change from session to session (Morgan-Lopez, Saavedra, Hien, \& Fals-Stewart, 2011).

Morgan-Lopez and Fals-Stewart (2008a) detailed four concerns that treatment researchers should consider regarding the choice of analytic strategy in modeling open enrollment group data: First, is there a relation between the probability of treatment group attendance and the (potential) substance use outcome (e.g., patient does not attend treatment because of relapse)? Second, do treatment effects vary by treatment attendance patterns? Third, do the subtypes of patients with regard to attendance patterns fluctuate from session to session? 
Fourth, are these fluctuations related to specific points of the calendar (e.g., holidays, seasons)? The extent to which causal inference is justified in the presence of any of these issues may depend on how well the analytic strategy handles each element (Morgan-Lopez $\&$ Fals-Stewart, 2006). Failure to consider these elements in rolling group data may obscure attendance class-specific treatment effects (Morgan-Lopez \& Fals-Stewart, 2007) as well as increase the likelihood of Type I errors (Morgan-Lopez \& Fals-Stewart, 2008b). Concerns about the impact of treatment attendance in and of itself may preclude the examination of overall treatment outcomes (irrespective of membership turnover) and may warrant a more nuanced approach to evaluating treatment efficacy and/or effectiveness (Feaster et al., 2003).

\section{Current Study}

The Women and Trauma Study, conducted within the National Institute on Drug Abuse National Drug Abuse Treatment Clinical Trials Network (NIDA CTN), is the largest multisite randomized clinical trial to date conducted with women with trauma histories in community substance abuse treatment. Design features of this trial were selected to maximize external validity while retaining important internal validity rigor. These features include (a) psychotherapy, Seeking Safety (Najavits, 2002), with prior evidence of efficacy and delivered by community treatment counselors (Hien et al., 2004) with high fidelity in an open enrollment, rolling group format; (b) an attention control group consisting of a health education curriculum (Women's Health Education; Miller, Pagan, \& Tross, 1998) of similar length; and (c) assessments, gathered consistently during the treatment period and 1 year after treatment, that included comprehensive documentation of service utilization.

Primary outcome analyses of the Women and Trauma Study followed an intent-to-treat approach, which applied hierarchical linear modeling techniques. Overall findings demonstrated that both group interventions led to significant reductions in PTSD symptoms during the in-treatment phase. Neither treatment, however, appeared to impact substance use outcomes differentially over the 1-year follow up period (Hien, Wells, et al., 2009). There was strong evidence for superiority of Seeking Safety over Women's Health Education in reducing risky sexual behavior among those at highest risk (Hien, Campbell, et al., 2010) and in reducing substance use among those who had significant changes in PTSD symptoms and were heavy users at baseline (Hien, Jiang, et al., 2010). The lack of more robust findings, specifically on substance use outcomes, suggests that standard statistical approaches, which did not account for varying attendance patterns and changing group membership, may have obscured the impact of the two treatments. Because the traumafocused treatment used in the trial was designed to address both PTSD and substance use outcomes directly and because regulating emotional distress in the trauma treatment should promote better ability to retain and apply the skills to improve coping with substances, further examination of the impact of attendance patterns and group membership turnover specifically on the substance use outcomes is warranted.

Furthermore, there have been advances in the theory of how treatment attendance (Barkham et al., 2006; Feaster et al., 2003) and group membership turnover (Morgan-Lopez \& FalsStewart, 2006, 2008a) impact substance use treatment outcomes and parallel advances in statistical methodology (Morgan-Lopez \& Fals-Stewart, 2007, 2008b) not previously considered in this area. Newer statistical techniques, such as latent class pattern mixture approaches (Lin, McCulloch, \& Rosenheck, 2004; Roy, 2003), can account for distinctive missingness/attendance patterns and group membership turnover (in addition to site-level variation). In the current investigation, we apply these new statistical methods to re-examine the specific impact of the Seeking Safety group therapy versus Women's Health Education from the Women and Trauma Study, taking into consideration attendance patterns and group 
membership turnover on substance use outcomes to further advance the treatments of cooccurring PTSD and substance use disorders. The following research questions are examined in this study: First, are there different treatment attendance patterns in this sample? Second, if so, does one of the patterns involve a titration of treatment frequently observed among individuals with PTSD and SUD? Third, are there differential substance use outcomes by treatment type associated with these attendance patterns? We further speculate that if an attempt to regulate emotional processes underlies a titrator attendance pattern, this pattern will be (a) more evident among those who received Seeking Safety compared with the Women's Health Education group and (b) significantly associated with better substance use outcomes, when compared with other attendance groups.

\section{Method}

\section{Participants}

This study used data collected from the NIDA CTN Women and Trauma Study, a randomized control trial comparing two group interventions for women with PTSD and SUD. Participants were 353 women enrolled in community-based, intensive, outpatient substance abuse treatment from seven geographically diverse programs in the United States.

Participants were eligible for the study if they reported at least one lifetime traumatic event and met Diagnostic and Statistical Manual of Mental Disorders (4th ed.; DSM-IV; American Psychiatric Association, 1994) criteria for either full or subthreshold PTSD in the past 30 days as assessed with the structured Clinician-Administered PTSD Scale (Blake et al., 1995). Subthreshold PTSD differed from full PTSD only in the number of symptom clusters that needed to be present; that is, participants had to meet Criterion B (reexperiencing the trauma) but had to meet only Criterion $\mathrm{C}$ (avoidance of trauma reminders) or Criterion D (hyperarousal) and not both. This is a commonly used definition of subthreshold PTSD (Blanchard, Hickling, Taylor, Loos, \& Gerardi, 1994; Grubaugh et al., 2005). Other inclusion criteria were (a) being a woman 18 to 65 years of age, (b) using alcohol or illicit substances within the past 6 months, and (c) meeting a current (within the prior year) diagnosis of drug or alcohol abuse or dependence. Women were excluded if they had (a) impaired mental cognition, as indicated by a Mini-Mental Status Exam (Folstein, Folstein, \& McHugh, 1975) score of less than 21, (b) significant risk of suicide or homicide in the prior 6 months as assessed with the Psychiatric Research Interview for Substance and Mental Disorders (Hasin, Trautman, \& Endicott, 1998), or (c) history of schizophreniaspectrum diagnosis or active (past 2 months) psychosis, as clinically assessed using the Composite International Diagnostic Interview for DSM-IV (Robins et al., 1988). Excluded participants were referred to clinical services at their treatment programs for more appropriate intervention.

Recruitment occurred over 21 months in 2004 and 2005. Female clients enrolled in the community treatment program were approached and informed of the study. If interested and willing, clients completed a brief eligibility assessment. Those meeting major eligibility criteria were then formally assessed in a two-part screening and baseline interview. After the baseline assessment, participants were randomized into either Seeking Safety or Women's Health Education treatment groups; each site received 60 sealed, tamper-evident, opaque envelopes generated by an independent statistician containing a randomization number corresponding to treatment assignment. Women completed brief, weekly assessments for substance use, PTSD symptoms, and service utilization during treatment and were reassessed with the full assessment battery 1 week, 3, 6, and 12 months posttreatment. Independent assessors administered baseline and follow-up assessments; they were unaware of study randomization, and participants were reminded before each follow-up assessment that the independent assessor should not have knowledge of their study condition. Informed 
consent was completed for the screening assessment, with study informed consent gathered prior to baseline. The study was approved by the institutional review board representing the coordinating site as well as at each individual treatment site. Table 1 includes information on demographics, baseline PTSD and SUD diagnoses, and traumatic experiences by treatment condition.

\section{Study Treatments}

Prior to attending groups, participants met individually with the group leader to receive an introduction to the treatment and review group rules. Treatment consisted of two 90-min sessions per week for 6 weeks. Groups ranged in size from two to eight women and operated with an open, rolling admission so that participants could enter the group at any point in the 12-session cycle (i.e., a participant might start with Session 7 and complete treatment with Session 6). Participants remained in study treatment regardless of attendance unless they missed four consecutive sessions with no study contact. Those who missed sessions were given the opportunity to review content by viewing a videotape of the missed group or groups, but this was not considered the same as attending a session. Participants were also terminated from study treatment if they withdrew from the treatment program.

Study treatments had different foci based on different theoretical orientations and hypothesized mechanisms of change (see the primary outcome article, Hien, Wells, et al., 2009 , for more on distinguishing among treatments). Briefly, the Seeking Safety treatment (Najavits, 2002) is a short-term manualized therapy (group or individual modality) utilizing cognitive behavioral strategies to reduce substance use and the negative impact of trauma exposure. Sessions are structured and include education on substance use disorders and PTSD, skills to prevent drug use and control PTSD symptoms, cognitive restructuring with particular attention to maladaptive thoughts associated with substance use and trauma symptoms, and a focus on building a healthy support network through the development of effective communication skills. Session topics are meaningfully connected to participant reports of both unsafe behavior and coping skills. The group format allows women to provide support and reinforcement to each other.

Women's Health Education (Miller et al., 1998) is a psychoeducational intervention that focuses on topics such as female anatomy, human sexual behavior, pregnancy and childbirth, nutrition, and diabetes. Women's Health Education provides equivalent facilitator attention, expectancy of benefit, and issue oriented focus but does not provide theory driven techniques, such as cognitive behavioral therapy and psychoeducation specific to substance abuse and PTSD. Topic presentations use minilecture, video, storytelling, and/or text readings as well as exercises to facilitate group discussion and application of materials.

\section{Treatment Fidelity}

Counselors and supervisors were selected from each site on the basis of willingness to be randomized to one of the two intervention conditions and demonstrated ability to deliver a manualized, cognitive behavioral style of therapy based on review of an audiotaped relapse prevention session. Following centralized training, counselors and supervisors were certified in the interventions after completing a training group of at least four sessions and meeting adequate adherence levels. During study implementation, all intervention sessions were videotaped, and approximately $50 \%$ were rated for adherence by local supervisors; counselors met weekly with supervisors. Intervention experts at the coordinating site corated a randomly selected quarter of the counselor session tapes reviewed by the supervisor to monitor reliability on adherence measure scores (see Hien, Wells, et al., 2009, for additional information on adherence measures and interrater reliability). 


\section{Measures}

Substance use-Information on alcohol and cocaine use was collected at each of the assessment time points, using the Addiction Severity Index (ASI-Lite, revised from the 1997 ASI, Fifth Edition; McLellan, Alterman, Cacciola, Metzger, \& O'Brien, 1992). Participants reported the number of days $(0-30)$ on which any of these substances were used. Because we were interested in treatment effects on any use, binary indicators were created at each time point from these measures. The ASI has demonstrated reliability and validity across diverse substance-using populations (Samet, Waxman, Hatzenbuehler, \& Hasin, 2007).

Additional measures-Measures that were beyond the scope of this study were also administered and included measures of sociodemographics, PTSD frequency, and PTSD intensity. These are discussed in Hien, Wells, et al. (2009).

\section{Data Analytic Approach}

As noted elsewhere (Morgan-Lopez \& Fals-Stewart, 2006, 2007, 2008a, 2008b), standard analytic approaches used for modeling treatment outcomes (e.g., repeated measures analysis of variance, group-clustered latent growth models) have limited utility in accounting for the changes over time in treatment group membership that occur within open enrollment groups (i.e., loss and addition of group members). Latent class pattern mixture modeling (LCPMM; Lin et al., 2004; Roy, 2003; see also Muthén, Jo, \& Brown, 2003) has been shown in both real (Morgan-Lopez \& Fals-Stewart, 2007) and simulated treatment outcome data (MorganLopez \& Fals-Stewart, 2008b) to be a promising approach for modeling specific nuances of the open enrollment group process (i.e., group membership turnover, latent attendance classes within treatment groups).

LCPMMs (a) account for variability in the treatment effect across a finite number of latent attendance classes among individuals in the same treatment group and (b) can be structured to model relations between attendance class membership and variation in patients' points of entry into treatment. The logic underlying the utility of LCPMMs to the analysis of data from substance abuse treatment trials with rolling/open enrollment groups is that, within any point of the trial, the proportions of different types of attendance patterns (and, therefore, different subtypes of patients) are allowed to vary at any given slice in time at which the trial is running (Morgan-Lopez \& Fals-Stewart, 2007, 2008a). Moreover, group composition and norms change over time in concert with changes in the membership of the group, which collectively may affect the efficacy of the treatment during particular periods of the history of the group (e.g., the proportion of dropouts decreases over time as the group becomes more cohesive and efficacious).

The focal point of the LCPMM model is a categorical latent variable (called Attend in Figure 1), which captures the estimated probabilities of membership in one of a finite number $(C)$ of latent classes (e.g., Attend $=1,2 \ldots C$ ). This variable represents multiple latent subpopulations within treatment outcome data, which vary between classes along three sets of variables (see Figure 1). First, classes can vary in their probabilities of treatment attendance at each session, through a series of binary indicators of whether each patient showed up for treatment at week $w(0=$ no show, $1=$ show $)$ beyond the initial session for each person. Second, differences may emerge on the distributions of the timing of treatment entry (i.e., Start Week), which (a) may show fluctuations over time in the proportions of patients from each subpopulation and (b) may be indicative of variation in the makeup of treatment groups that is dependent on calendar time (Morgan-Lopez \& FalsStewart, 2007, 2008b). Finally, differences across the classes may emerge on the effect of the treatment condition (Seeking Safety/Women's Health Education) on growth over time in the outcome for the in-treatment interval $\left(\beta_{\mathrm{ITI}}\right)$ and posttreatment interval $\left(\beta_{\mathrm{PTI}}\right)$ slopes that 
underlie the substance use outcome measures ( $y_{\text {baseline }}-y_{12}$ month $)$; differences in the intercept $\left(\mathrm{a}_{\mathrm{ITI}}\right)$ by treatment condition are also possible, though they would, of course, be indicative of undesirable differences at baseline.

If there is more than one class, the growth parameters (i.e., conditional growth parameter means, treatment effects) from each class are averaged and weighted by the class proportions, and standard errors are calculated using the delta method (see Hedeker \& Gibbons, 1997, pp. 74 -76; Morgan-Lopez \& Fals-Stewart, 2007, p. 593) in order to estimate the overall treatment effect; note that this weighted averaging can now be conducted within a single step within an LCPMM analysis (e.g., with the Model Constraint command in Mplus v5.2). Both overall and class-specific treatment effects are presented here.

\section{Results}

\section{Preliminary Identification of Functional Form}

The group stratified finite mixture modeling facilities within Mplus 5 (Muthén \& Muthén, 1998 -2009) were used for all analyses under robust maximum likelihood estimation (i.e., Mplus MLR estimation under stratification; Asparouhov, 2005). Prior to fitting the models of interest, the observed proportions for alcohol and cocaine use across preintervention and the four follow-up assessments were plotted to get a sense of the optimal functional form; assessing the correct functional form (i.e., latent variable structure) a priori also minimizes the likelihood of overextraction of latent classes (Bauer \& Curran, 2004). We concluded, on the basis of the graphical analyses, that the optimal functional form for the two substances was piecewise linear with two distinct periods of growth: (a) growth from preintervention to 1-week posttreatment follow-up and (b) growth from 1-week through 12-month posttreatment follow-up. The time steps for each of these three growth parameters (intercept, first slope, second slope) were structured such that the intercept was set at preintervention (i.e., estimated level probability of use in the week prior to the participant's first treatment session).

Graphical analyses were supplemented by a series of unconditional growth models (with probit link functions for categorical outcomes for this and all subsequent analyses) examined under alternate functional forms. When we used chi-square difference testing, the fit of the unconditional two-piece piecewise linear model described earlier did not differ significantly from unconditional models that included a quadratic growth factor for growth during the posttreatment phase $\left(\Delta \chi^{2}\right.$ for both substances was at or exceeded $\left.p=.10\right)$. Thus, the twopiece piecewise linear functional form was used for all subsequent analyses.

\section{Identification of the Optimal Number of Attendance Classes}

Next, a series of two-piece linear probit LCPMMs (under group stratification for nonindependence because of site-level clustering), with varying numbers of latent attendance classes, was fit to determine the optimal number of classes. Bayesian information criterion (BIC) and entropy were used to determine the optimal number of classes. As shown in Table 2, the BIC suggests three classes are optimal across each of the substances (from the perspective of model fit); however, the two-class solutions produced entropy values that were best. We chose the three-class solution(s) because BIC is more closely tied to model fit (i.e., omission of classes) than is entropy (classification quality), though these measures tend to agree in practice. 


\section{Three-Class Models}

Probabilities of treatment attendance across classes-Across the two sets- one for alcohol use, one for cocaine use- of three-class LCPMM analyses, a similar pattern emerged for treatment attendance (only patterns from the alcohol model are shown, to avoid redundancy). The first class, completers, never decreased below an $80 \%$ probability of treatment attendance; the class membership (based on posterior probabilities of class membership as opposed to the most likely class assignment) was between $47 \%$ and $48 \%$ of the total sample across both substances. The second class under the three-class models is called droppers. This class never exceeded a $41 \%$ probability of attending treatment, and by the fourth session they would have attended, they had virtually 0 probability of attendance for the remainder of the protocol; this class made up around $24 \%$ of the sample across all substances/models. The third class is referred to as the titrators class; through the seventh session, these patients ranged between a $50 \%$ and an $80 \%$ probability of attending treatment, with further decreases in the probability of showing up for treatment across sessions but with probabilities that never approached 0 as in the droppers class; this class ranged between $27 \%$ and $28 \%$ of the sample across the substances/models. In both LCPMM analyses, treatment condition was not predictive of attendance class membership such that the proportion of completers, titrators, and droppers did not differ significantly for the Seeking Safety condition or the Women's Health Education condition (e.g., $p$ values were all greater than .17).

Across the classes, pretreatment differences among participants along sociodemographic and clinical variables, including age, race, education, symptoms of depression, paranoid ideation, and 12-step affiliation, were nonsignificant at the individual level as estimated in separate mixture models. However, at the group level (i.e., variation in the percentages of patients within each class across treatment groups; $N=12$ treatment groups), groups with higher proportions of (eventual) dropouts had greater group mean levels of baseline psychiatric problems $(p=.013)$ and family problems $(p=.023)$. Conversely, treatment groups with higher proportions of (eventual) titrators had lower group mean levels of psychiatric problems $(p=.002)$, family problems $(p=.09)$, depression $(p<.001)$, and paranoid ideation $(p=.07){ }^{1}$

Distributions of the month of trial entry-The histogram (alcohol model only) in Figure 2 shows fluctuation in the number of patients within each class in the three-class model(s) throughout the span of the trial across 22 months. As noted earlier, the overall split in class membership was around $48 \%$ completers, $24 \%$ droppers, and $28 \%$ titrators based on the sums of posterior probabilities of class membership. Prior to Month 10, droppers made up 26\% of the sample, but they made up only $18 \%$ of the sample beyond Month 10, suggesting lower dropout probabilities during the second half of the trial; conversely, titrators made up $21 \%$ of the sample from Months 1 through 10 and increased to $26 \%$ beyond Month 10, which may be suggestive of greater treatment retention as the trial moved forward in calendar time.

\section{Effect of Attendance Class and Treatment on Alcohol Use (See Table 3 and Figure 3)}

Completers-Among completers, there were significant decreases in the probability of alcohol use (as evidenced by logistic regression coefficients) from baseline to 1-week posttreatment follow-up, $b=-1.334(0.542), t=-2.463, p=.014$, followed by nonsignificant increases in alcohol use from 1-week through 12-month follow-up, $b=0.215(0.744), t=$

\footnotetext{
${ }^{1}$ Psychiatric and family problems were assessed using composite scores compiled from two domains on the ASI-Lite (McLellan et al., 1992). Depression and paranoid ideation symptom scores were derived from subscales on the Brief Symptom Inventory (Derogatis, 1993).
} 
$0.289, p=.77$. Neither the in-treatment nor the posttreatment slopes $(p=.26$ and $p=.09$, respectively) differed between completers in the Seeking Safety condition and the Women's Health Education condition.

Droppers-Among droppers, there were nonsignificant increases in alcohol use from baseline to 1 -week follow-up, $b=0.446(1.210), t=0.368, p=.71$, followed by nonsignificant increases in alcohol use from 1-week through 12-month follow-up, $b=0.119$ (0.815), $t=0.146, p=.88$. Neither the in-treatment nor the posttreatment slopes $(p=.64$ and $p=.92$, respectively) differed between droppers in the Seeking Safety condition and the Women's Health Education condition.

Titrators-Among titrators, there were nonsignificant decreases in alcohol use from baseline to 1-week follow-up, $b=-1.531$ (1.056), $t=-1.449, p=.14$, followed by nonsignificant increases in alcohol use from 1-week through 12-month follow-up, $b=0.463$ (0.740), $t=0.626, p=.53$. Although the in-treatment slopes did not differ between Seeking Safety and Women's Health Education $(p=.94)$, patients in Seeking Safety had lower rates of alcohol use from 1-week through 12-month follow-up compared with Women's Health Education, $b=-0.203$ (0.085), $t=-2.389, p=.017$.

Effects averaged across classes-When combined across classes, no differences in treatment effects on alcohol use were observed between Seeking Safety and Women's Health Education. Contrasts were conducted to assess whether treatment effect differed across classes (e.g., treatment effect for completers vs. droppers); none of those contrasts were significantly different from 0 (all $p s>.65$ ).

\section{Effect of Attendance Class and Treatment on Cocaine Use: All Classes}

Among all classes, nonsignificant decreases in cocaine use were observed during the intreatment and posttreatment phase; these decreases did not differ between Seeking Safety and Women's Health Education.

\section{Discussion}

The extant literature has produced a limited number of multisite effectiveness trials that consider the influence of attendance patterns and membership turnover on outcomes for group psychotherapy with rolling admission. The present study took important initial steps to address this gap through a secondary analysis of recently published clinical trial data (Hien, Wells, et al., 2009), using innovative statistical approaches to examine the impact of attendance patterns and more accurately model rolling admissions groups on in-treatment and posttreatment substance use outcomes. We were interested in drawing from an emotional regulation perspective of PTSD and SUD to examine whether varying patterns of treatment attendance would reveal treatment-specific differences in outcomes on substance use over time.

With respect to the first research questions, findings are consistent with three treatment attendance patterns distinct by their probabilities of session attendance across 6 weeks of treatment (Figure 4). Specifically, across our heterogeneous multisite sample, three different attendance patterns were identified: patients who tended to have at least an $80 \%$ probability of attending sessions (completers), participants who tended to discontinue treatment by the fourth session (droppers), and patients whose tendency was to have a high probability of attending group through their seventh session but then showed sporadic attendance thereafter (titrators). 
Thus, with respect to the second research question, there does appear to be a class of participants whose attendance is consistent with titration of treatment, that is, participants who have significant numbers of missed sessions but do not appear to fully terminate treatment engagement. With respect to the third research question, when attendance patterns were jointly modeled with substance use outcome trajectories in this secondary analyses, important differential treatment effects emerged that were previously undetected. As hypothesized, benefits for the trauma-focused treatment were most evident among the titrators class. Titrating patients showed reductions over time in substance use outcomes in the Seeking Safety condition compared with Women's Health Education, a finding that had not been observed using conventional longitudinal modeling methods (e.g., hierarchical linear models) for the substance use outcomes (Hien, Wells, et al., 2009).

Notably, however, although findings for cocaine were in a similar direction, only alcohol use outcomes reached a level of statistical significance. Our findings underscore the importance of considering dose-response (albeit latent in this case) and treatment group composition to better identify for whom and to what degree a specific treatment might work (Stulz, Lutz, Leach, Lucock, \& Barkham, 2007). Results suggest that the number of sessions attended might not be as useful as the quality of the participation for this PTSD and SUD population. For titrators who received Seeking Safety, attendance through the seventh session might have been an optimal or good enough level in terms of dose responsiveness (Barkham et al., 2006). Further, they appeared to be able to apply what they learned in treatment over a 12-month follow-up period. Conversely, there was no evidence to suggest that those who attended and received more Seeking Safety sessions derived additional benefit from the greater dose of therapy. This is also consistent with a relatively large body of research indicating that the "level of improvement and treatment duration are mutually regulated so that treatments tend to end when clients, on average, have improved to a degree or level that is good enough" (Barkham et al., 2006, p. 161). Relatedly, in one of the earliest studies of Seeking Safety among women outpatients, Najavits et al. (1998) found that treatment completers were actually more impaired than dropouts and had a worsening of somatic symptoms posttreatment, suggesting that treatment completion may not always be beneficial.

Of note, results comparing titrators and completers revealed no statistically significant differences on any individual level severity (PTSD or substance use disorders) or demographic correlates. That is, neither titrators nor completers appeared to be more severely impaired. However, further analyses of the group context did detect relevant differences. Specifically, treatment groups with a larger proportion of titrators had members with more medical problems but also lower baseline levels of anxiety, depression, and selfreported symptoms of paranoia compared with groups made up of more completers. Thus, we could also speculate that members of titrator groups might have had more barriers to attendance (e.g., more medical problems) but, when attending treatment, were in groups with other patients who had less severe psychopathology and were overall more able to profit from the treatment content and process.

Among those in the Women's Health Education groups, completers derived a similar benefit on alcohol outcomes to the titrators in the Seeking Safety groups. This treatment effect suggests that the type of treatment offered in the group context also differentially affects outcomes when group membership characteristics are considered. Among those receiving a non-trauma-focused treatment, even when groups had more psychiatric comorbidity, there was still a benefit of the group therapy for substance outcomes for these participants. This finding suggests that important nonspecific therapeutic factors, such as support, consistency, and a safe therapeutic environment (Moos, 2007; without the added trauma content), were differentially beneficial for these patients in terms of alcohol use outcomes. It is also 
possible that the psychoeducational component of the health education group had specific therapeutic effects on alcohol use outcomes. Studies indicate that, given the deficits in selfcare often evident in individuals with substance use disorders, the provision of accurate and comprehensible information about specific health issues can lead to positive treatment outcomes (Cummings \& Cummings, 2008; La Salvia, 1993).

Across groups and membership patterns, only one of the groups during the in-treatment phase differed from the others. Among those in the Women's Health Education groups with more droppers, alcohol outcomes were significantly worse than those of all other groups, whose alcohol use diminished over the treatment phase. In light of the fact these groups did receive substance abuse coping skills, it is not surprising that those who dropped out of treatment early and received few-to-no sessions of any skill-based approach to managing substance use would have poorer outcomes overall. Because our study was the first to begin disentangling attendance-specific effects for an active treatment and active comparison, subsequent studies are needed to dismantle these effects.

\section{Limitations}

The present study had certain limitations, and it is important to be cautious in interpreting the results. First, additional service utilization was not taken into account in this analysis. Similarly, although we have presented elsewhere that PTSD improvements were differentially associated with substance use reductions among more severely ill participants (Hien, Jiang, et al., 2010), this analysis did not incorporate PTSD symptom changes or directionality in comorbidity (i.e., primary vs. secondary PTSD), as the complexity of that analysis was beyond the scope of this article. Also, because we did not directly measure emotional regulation, we can only speculate that titrators' intermittent attendance was related to attempts to adaptively manage emotional distress or activation and that better regulation in this group may have led to superior outcomes when receiving the trauma therapy. For example, an alternative explanation for the pattern of attendance among the titrators could be a result of participant's perception of value of the treatment, but this was not measured and so could not be determined. Although completers appeared not to benefit further with regard to alcohol or cocaine use than the titrators, there may have been other factors that were neither measured nor analyzed that induced completers to receive the maximum dose that could help to explain this finding. Another measurement limitation of note is that substance use outcomes were based on self-report and did not account for changes in quantity of use (e.g., standard drink units). Finally, because this was a voluntary study, not all eligible participants chose to enroll, reducing generalizability of results.

Limitations notwithstanding, there are several strengths of the present study, which make important contributions to the literature. These include our use of a heterogeneous patient population, use of a relatively large sample, and analytic provisions to account for site-level variation, group membership turnover, and consideration of treatment dose in analyses. This analysis expanded the addiction measures beyond those used in the primary outcome article (Hien, Wells, et al., 2009) to include substance-specific use outcomes from the ASI-Lite, common in addiction studies.

\section{Implications and Future Directions}

This reanalysis of the Women and Trauma Study randomized multisite trial provides a new model for considering how to account for participant-driven attendance patterns and rolling groups, both in analysis and interpretation of treatment results. It specifically lends support to the importance of considering emotion regulatory processes and treatment titration among those with PTSD and SUD. Those interested in translational research, which in part examines how psychotherapeutic treatments fare in community-based settings, must 
consider a priori these kinds of analyses in order to illuminate potentially subtle yet clinically relevant differences based on how a patient attends a treatment and who else is attending with them. Future studies should attend to specific design issues to account for site-specific factors that may influence outcomes associated with delivery of treatment, as well as assess the effect of concurrent service utilization. Because of the rolling group design, we selected 12 sessions of Seeking Safety to be free standing, assuming that session content is independent from treatment dose. However, it is quite possible that order of sessions received may also impact treatment efficacy. Future research should focus on this complex analysis in fuller detail.

Beyond implications for research, findings suggest potentially valuable directions to improve standard of care for women with PTSD and comorbid SUD. In line with the concepts of personalized medicine, our findings underscore the importance of individualized treatment planning versus standardized decisions about treatment attendance, treatment duration, and primary substance of abuse within community-based substance abuse recovery programs. Treatment programs may benefit from lessening the emphasis on very high attendance rates and target interventions and program policies that recognize that attendance rates of $50 \%$ to $80 \%$ may confer equal or even greater benefit. More fundamentally, these study findings should prompt providers to consider that female patients with comorbid PTSD and SUD may be in the best position to determine when, how often, or which sessions to attend; such perspectives might not only be empowering to patients but might be efficacious as well.

\section{Acknowledgments}

We acknowledge the work and support of the six regional research training centers involved in the implementation of this study and the invaluable commitment of the patients and staff at the seven participating community-based treatment programs. Staff from the National Institute on Drug Abuse's Clinical Trials Network collaborated in the design and conduct of the study, assisted with data management and quality assurance during the trial, and provided comments for consideration on this article. Portions of this research were presented at the American Psychological Association's annual meeting in San Diego, California (August 2010) and at the Second International Congress on Dual Disorders in Barcelona, Spain (October 2011). The research reported in this article was supported by the following grants from the National Institute on Drug Abuse: U10DA13035 (to Edward V. Nunes, principal investigator [PI]), U10 DA13714 (to Dennis Donovan, PI), U10 DA13038 (to Kathleen Carroll, PI), U10 DA13732 (to Eugene Somoza, PI), U10 DA13727 (to Kathleen Brady, PI), U10 DA013720 (to Jose Szapocznik, PI), U10 DA013046 (to John Rotrosen, PI), K24 DA022412 (to Edward V. Nunes), R01DA025198 (to Antonio Morgan Lopez), and R01DA025198 (to Lissette Saavedra). The clinical trial identification number is NCT00078156 (National Institute on Drug Abuse). Edward V. Nunes has served on the advisory panel and speakers' bureau of Alkermes/Cephalon, Inc. (resigned October 2007).

\section{References}

American Psychiatric Association. Diagnostic and statistical manual of mental disorders. 4. Washington, DC: Author; 1994.

Asparouhov T. Sampling weights in latent variable modeling. Structural Equation Modeling. 2005; 12:411-434.10.1207/s15328007sem1203_4

Barkham M, Connell J, Stiles WB, Miles JNV, Margison F, Evans C, Mellor-Clark J. Dose-effect relations and responsive regulation of treatment duration: The good enough level. Journal of Consulting and Clinical Psychology. 2006; 74:160-167.10.1037/0022-006X.74.1.160 [PubMed: 16551153]

Barkham M, Rees A, Shapiro DA, Stiles WB, Agnew RM, Halstead J, Harrington VMG. Outcomes of time-limited psychotherapy in applied settings: Replication of the second Sheffield Psychotherapy Project. Journal of Consulting and Clinical Psychology. 1996; 64:1079-1085.10.1037/0022-006X. 64.5.1079 [PubMed: 8916639] 
Bauer DJ, Curran PJ. The integration of continuous and discrete latent variable models: Potential problems and promising opportunities. Psychological Methods. 2004; 9:3-29.10.1037/1082-989X. 9.1.3 [PubMed: 15053717]

Blake DB, Weathers FW, Nagy LM, Kaloupek DG, Gusman FD, Charney DS, Keane TM. The development of a Clinician-Administered PTSD Scale. Journal of Traumatic Stress. 1995; 8:7590.10.1002/jts.2490080106 [PubMed: 7712061]

Blanchard EB, Hickling EJ, Taylor AE, Loos WR, Gerardi RJ. Psychological morbidity associated with motor vehicle accidents. Behavior Research and Therapy. 1994; 32:283290.10.1016/0005-7967(94)90123-6

Brady K. Comorbid posttraumatic stress disorder and substance use disorders. Psychiatric Annals. 2001; 31:313-319.

Bryk, A.; Raudenbush, SW. Hierarchical linear models. Thousand Oaks, CA: Sage; 1992.

Cummings, NA.; Cummings, JL. Psychoeducation in conjunction with psychotherapy practice. In: O'Donohue, W.; Cummings, NA., editors. Evidence-based adjunctive treatments. New York, NY: Academic Press; 2008. p. 41-59.

Dansky BS, Saladin ME, Brady KT, Kilpatrick DG, Resnick HS. Prevalence of victimization and posttraumatic stress disorder among women with substance use disorders: Comparison of telephone and in-person assessment samples. International Journal of the Addictions. 1995; 30:1079-1099. [PubMed: 7591350]

Derogatis, LR. Brief Symptom Inventory: Administration, scoring, and procedures manual. Minneapolis, MN: National Computer Systems; 1993.

Feaster DJ, Newman F, Rice C. Longitudinal analysis when the experimenter does not determine when treatment ends: What is dose-response? Clinical Psychology \& Psychotherapy. 2003; 10:352360.10.1002/cpp.382 [PubMed: 16724159]

Folstein MF, Folstein S, McHugh P. "Mini-Mental State": A practical method for grading the cognitive state of patients for the clinician. Journal of Psychiatric Research. 1975; 12:189-198. [PubMed: 1202204]

Greenfield SF, Brooks AJ, Gordon SM, Green CA, Kropp F, McHugh RK, Miele GM. Substance abuse treatment entry, retention, and outcome in women: A review of the literature. Drug and Alcohol Dependence. 2007; 86:1-21.10.1016/j.drugalcdep.2006.05.012 [PubMed: 16759822]

Grubaugh AL, Magruder KM, Waldorp AE, Elhai JD, Knapp RG, Frueh BC. Subthreshold PTSD in primary care: Prevalence, psychiatric disorders, healthcare use, and functional status. Journal of Nervous and Mental Disease. 2005; 193:658-664.10.1097/01.nmd.0000180740.02644.ab [PubMed: 16208161]

Hasin D, Trautman K, Endicott J. Psychiatric Research Interview for Substance and Mental Disorders: Phenomenologically-based diagnosis in patients who abuse alcohol or drugs. Psychopharmacology Bulletin. 1998; 34:3-8. [PubMed: 9564191]

Hedeker D, Gibbons RD. Application of random-effects pattern-mixture models for missing data in longitudinal studies. Psychological Methods. 1997; 2:64-78.10.1037/1082-989X.2.1.64

Hien DA, Campbell A, Killeen T, Hu M, Hansen C, Jiang H, Nunes EV. The impact of traumafocused group therapy upon HIV risk behaviors in the NIDA Clinical Trials Network "Women and Trauma" multi-site study. AIDS and Behavior. 2010; 14:421-430.10.1007/s10461-009-9573-7 [PubMed: 19452271]

Hien DA, Cohen LR, Campbell ANC. Methodological innovation to increase the utility and efficiency of psychotherapy research for patients with co-occurring mental health and substance use disorders. Professional Psychology: Research and Practice. 2009; 40:502-509.10.1037/a0014966

Hien DA, Cohen LR, Miele GM, Litt LC, Capstick C. Promising treatments for women with comorbid PTSD and substance use disorders. American Journal of Psychiatry. 2004; 161:14261432.10.1176/appi.ajp.161.8.1426 [PubMed: 15285969]

Hien DA, Jiang H, Campbell ANC, Hu M, Miele GM, Cohen LR, Nunes EV. Do treatment improvements in PTSD severity affect substance use outcomes? A secondary analysis from a randomized clinical trial in NIDA's Clinical Trials Network. American Journal of Psychiatry. 2010; 167:95-101.10.1176/appi.ajp.2009.09091261 [PubMed: 19917596] 
Hien DA, Wells E, Jiang H, Suarez-Morales L, Campbell A, Cohen L, Nunes EV. Multisite randomized trial of behavioral interventions for women with co-occurring PTSD and substance use disorders. Journal of Consulting and Clinical Psychology. 2009; 77:607-619.10.1037/ a0016227 [PubMed: 19634955]

Lambert MJ, Hansen NB, Finch AE. Patient focused research: Using patient outcome data to enhance treatment effects. Journal of Consulting and Clinical Psychology. 2001; 69:159172.10.1037/0022-006X.69.2.159 [PubMed: 11393594]

La Salvia TA. Enhancing addiction treatment through psychoeducational groups. Journal of Substance Abuse Treatment. 1993; 10:439-444.10.1016/0740-5472(93)90003-K [PubMed: 8246317]

Lin H, McCulloch CE, Rosenheck RA. Latent pattern mixture model for informative intermittent missing data in longitudinal studies. Biometrics. 2004; 60:295-305.10.1111/j.0006-341X. 2004.00173.x [PubMed: 15180654]

Lincoln A, Liebschultz J, Chernoff M, Nguyen D, Amaro H. Brief screening for co-occurring disorders among women entering substance abuse treatment. Substance Abuse Treatment and Prevention Policy. 2006; 1(26)10.1186/1747-597X-1-26

McLellan AT, Alterman AI, Cacciola J, Metzger D, O'Brien CP. A new measure of substance abuse treatment: Initial studies of the treatment services review. Journal of Nervous and Mental Disease. 1992; 180:101-110.10.1097/00005053-199202000-00007 [PubMed: 1737971]

Miller, S.; Pagan, D.; Tross, S. Unpublished treatment manuscript. Columbia University; New York: 1998. Women's Health Education: Peer activism for female partners of injection drug users.

Mills KL, Lynskey M, Teesson M, Ross J, Dark S. Post-traumatic stress disorder among people with heroin dependence in the Australian treatment outcome study (ATOS): Prevalence and correlates. Drug and Alcohol Dependence. 2005; 77:243-249.10.1016/j.drugalcdep.2004.08.016 [PubMed: 15734224]

Moos RH. Theory-based active ingredients of effective treatments for substance use disorders. Drug and Alcohol Dependence. 2007; 88:109-121.10.1016/j.drugalcdep.2006.10.010 [PubMed: 17129682]

Morgan-Lopez AA, Fals-Stewart W. Analytic complexities associated with group therapy in substance abuse treatment research: Problems, recommendations, and future directions. Experimental and Clinical Psychopharmacology. 2006; 14:265-273.10.1037/1064-1297.14.2.265 [PubMed: 16756430]

Morgan-Lopez AA, Fals-Stewart W. Analytic methods for modeling longitudinal data from rolling therapy groups with membership turnover. Journal of Consulting and Clinical Psychology. 2007; 75:580-593.10.1037/0022-006X.75.4.580 [PubMed: 17663612]

Morgan-Lopez AA, Fals-Stewart W. Analyzing data from open enrollment groups: Current considerations and future directions. Journal of Substance Abuse Treatment. 2008a; 35:3640.10.1016/j.jsat.2007.08.005 [PubMed: 17936551]

Morgan-Lopez AA, Fals-Stewart W. Consequences of misspecifying the number of latent treatment attendance classes in modeling group membership turnover within ecologically-valid behavioral treatment trials. Journal of Substance Abuse Treatment. 2008b; 35:396-409.10.1016/j.jsat. 2008.03.002 [PubMed: 18513917]

Morgan-Lopez AA, Saavedra LM, Hien DA, Fals-Stewart W. Estimating statistical power for open enrollment group treatment trials. Journal of Substance Abuse Treatment. 2011; 40:3-17.10.1016/ j.jsat.2010.07.010 [PubMed: 20832971]

Muthén BO, Jo B, Brown CH. Comment on the Barnard, Frangakis, Hill, \& Rubin article, "Principal stratification approach to broken randomized experiments: A case study of school choice vouchers in New York City. Journal of the American Statistical Association. 2003; 98:311-314.

Muthén, LK.; Muthén, BO. Mplus user's guide. Los Angeles, CA: Authors; 1998-2009. (Version 4)

Najavits, LM. Seeking Safety: A treatment manual for PTSD and substance abuse. New York, NY: Guilford; 2002.

Najavits LM, Weiss RD, Shaw SR. The link between substance abuse and posttraumatic stress disorder in women: A research review. American Journal on Addictions. 1997; 6:273-283. [PubMed: 9398925] 
Najavits LM, Weiss RD, Shaw SR, Muenz L. "Seeking Safety": Outcome of a new cognitivebehavioral psychotherapy for women with posttraumatic stress disorder and substance dependence. Journal of Traumatic Stress. 1998; 11:437-456.10.1023/A:1024496427434 [PubMed: 9690186]

Robins LN, Wing J, Wittchen HU, Helzer JE, Babor TF, Burke J, Towle LH. The Composite International Diagnostic Interview: An epidemiologic instrument suitable for use in conjunction with different diagnostic systems and in different cultures. Archives of General Psychiatry. 1988; 45:1069-1077. [PubMed: 2848472]

Roth S, Newman E, Pelcovitz D, van der Kolk B, Mandel F. Complex PTSD in victims exposed to sexual and physical abuse: Results from the $D S M-I V$ field trial for posttraumatic stress disorder. Journal of Traumatic Stress. 1997; 10:539-555.10.1002/jts.2490100403 [PubMed: 9391940]

Roy J. Modeling longitudinal data with nonignorable dropouts using a latent dropout class model. Biometrics. 2003; 59:829-836.10.1111/j.0006-341X.2003.00097.x [PubMed: 14969461]

Samet S, Waxman R, Hatzenbuehler M, Hasin DS. Assessing addiction: Concepts and instruments. Addiction Science and Clinical Practice. 2007; 4:19-31.10.1151/ascp074119 [PubMed: 18292706]

Stewart, SH.; Ouimette, P.; Brown, PJ. Gender and the co-morbidity of PTSD with substance use disorders. In: Kimmerling, R.; Ouimette, P.; Wolfe, J., editors. Gender and PTSD. New York, NY: Guilford Press; 2002. p. 232-270.

Stulz N, Lutz W, Leach C, Lucock M, Barkham M. Shapes of early change in psychotherapy under routine outpatient conditions. Journal of Consulting and Clinical Psychology. 2007; 75:864874.10.1037/0022-006X.75.6.864 [PubMed: 18085904]

Swift JK, Callahan JL, Levine JC. Using clinically significant change to identify premature termination. Psychotherapy: Theory, Research, Practice, Training. 2009; 46:328-335.10.1037/ a0017003

Triffleman E. Gender differences in a controlled pilot study of psychosocial treatments in substance dependent patients with post-traumatic stress disorder: Design considerations and outcomes. Alcoholism Treatment Quarterly. 2000; 18:113-126.10.1300/J020v18n03_10

Weiss RD, Jaffe WB, de Menil VP, Cogley CB. Group therapy for substance use disorders: What do we know? Harvard Review of Psychiatry. 2004; 12:339-350.10.1080/10673220490905723 [PubMed: 15764469]

\section{Appendix}

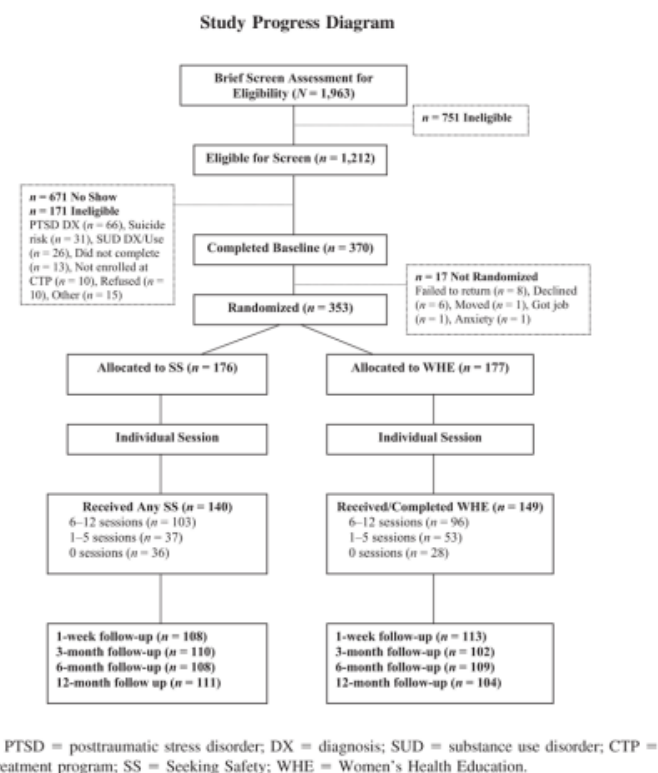

J Consult Clin Psychol. Author manuscript; available in PMC 2013 June 14. 


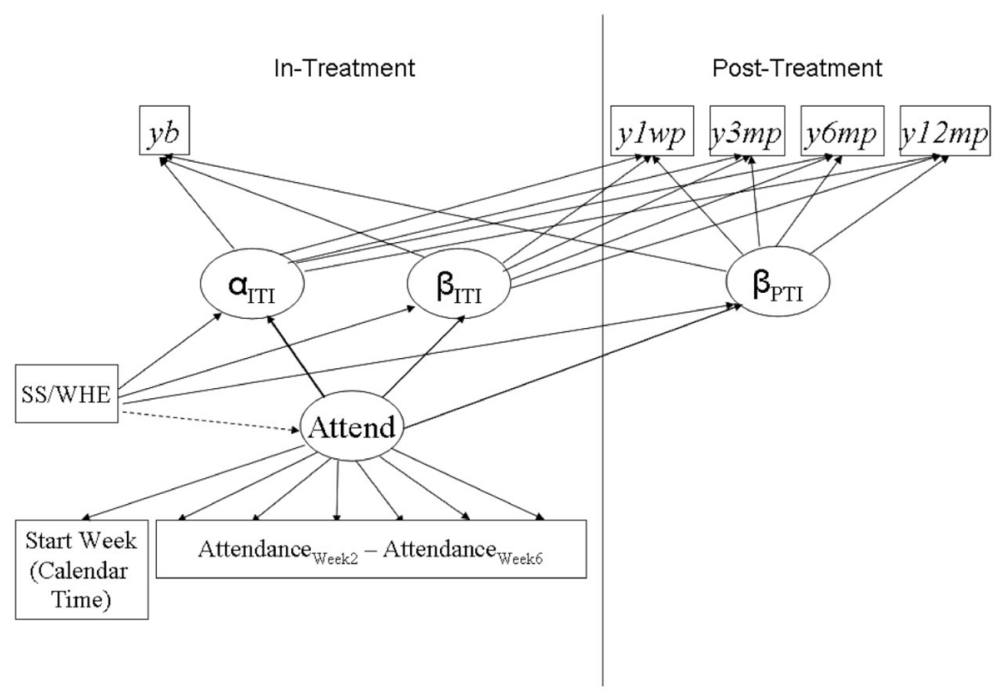

Figure 1.

Latent class pattern mixture model: $y b-y 12 \mathrm{mp}=$ Binary indicators of substance use from baseline through 12-month follow-up; SS/WHE = treatment condition (Seeking Safety = 1; Women's Health Education $=0$ ); $a_{\text {ITI }}=$ estimated level of the outcome at time $=0 ; \beta_{\text {ITI }}=$ estimated rate of change from baseline to immediate posttreatment (in-treatment interval); $\beta_{\mathrm{PTI}}=$ estimated rate of change from immediate posttest through 1-year follow-up. 


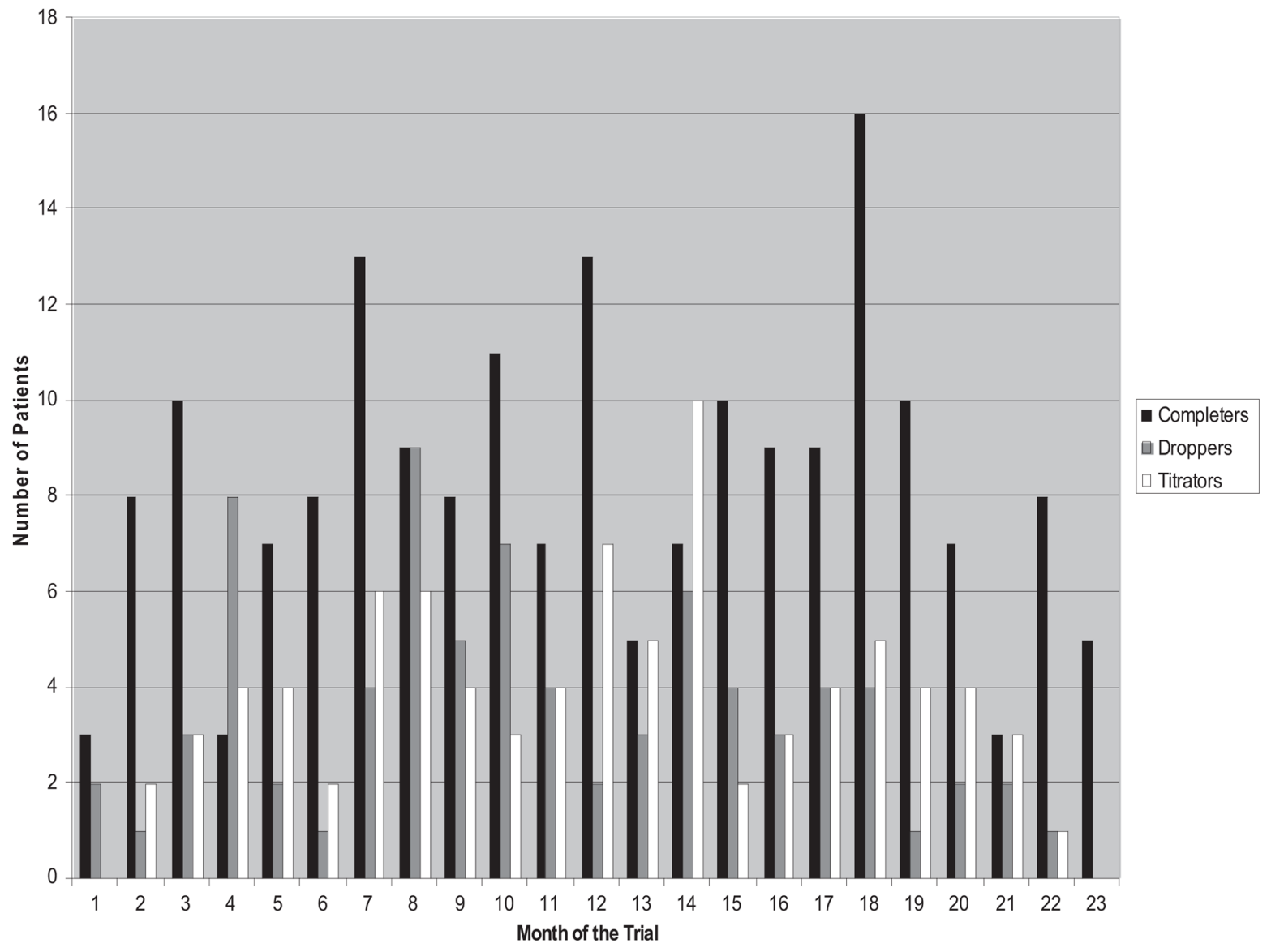

Figure 2.

Latent class membership histogram across calendar time. 


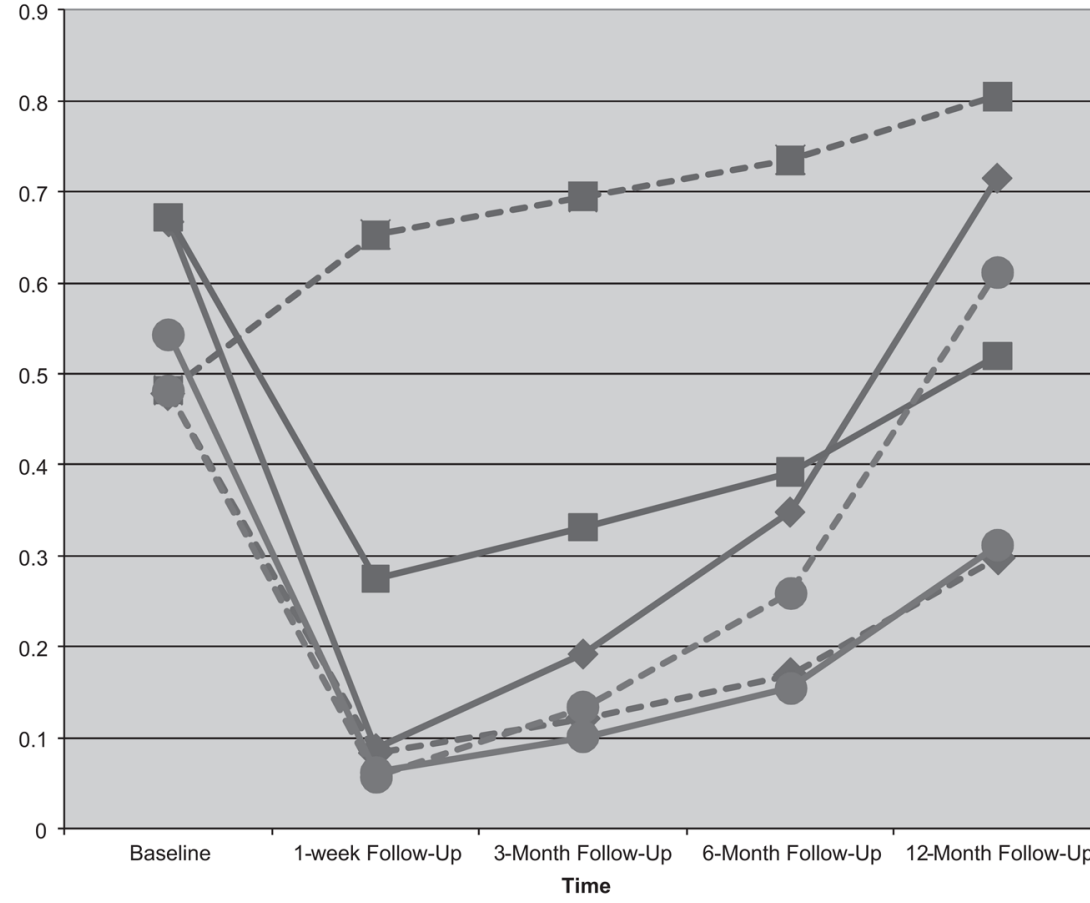

\begin{tabular}{|l|}
\hline- SS Completers \\
$-\infty-$ WHE Completers \\
- SS Droppers \\
- -WHE Droppers \\
- SS Titrators \\
$-\infty$ WHE Titrators
\end{tabular}

Figure 3.

Predicted probability of alcohol use over time by treatment group (Seeking Safety or Women's Health Education) and latent class membership. 


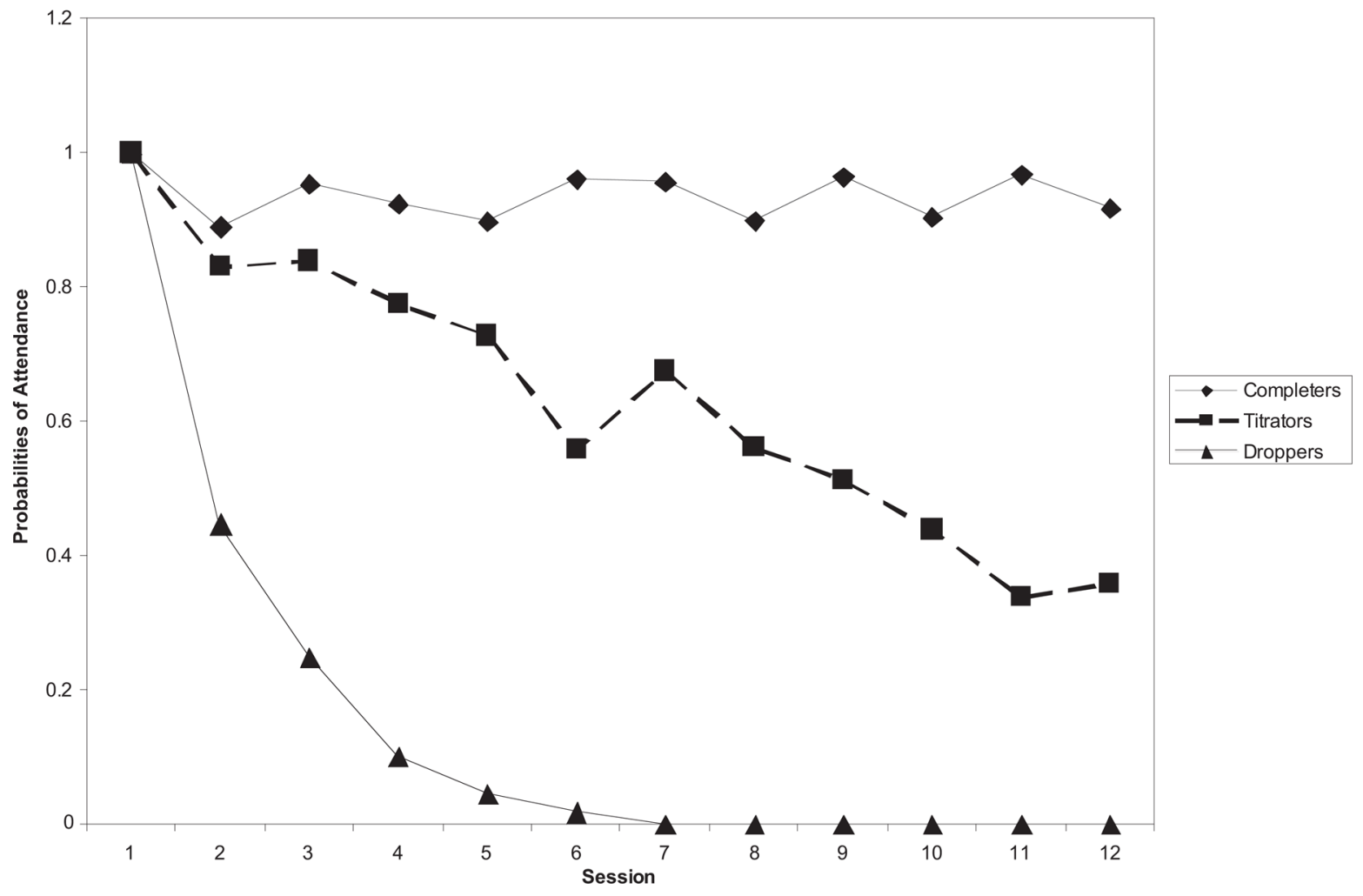

Figure 4.

Attendance probabilities by latent class. 
Table 1

Baseline Participant and Diagnostic Characteristics by Treatment Group ( $=353)$

\begin{tabular}{|c|c|c|c|}
\hline Variable & Total & Seeking Safety ${ }^{a}(N=176)$ & Women's Health Education $^{a}(N=177)$ \\
\hline Age (years) & $39.2(9.3)$ & $39.3(9.5)$ & $39.0(9.1)$ \\
\hline \multicolumn{4}{|l|}{ Race/ethnicity } \\
\hline African American/Black & 34.0 & 33.0 & 35.0 \\
\hline Caucasian & 45.6 & 47.2 & 44.1 \\
\hline Latina & 6.5 & 4.0 & 9.0 \\
\hline Multiracial/other & 13.9 & 15.9 & 11.9 \\
\hline Education (years) & $12.5(2.4)$ & $12.7(2.3)$ & $12.4(2.6)$ \\
\hline \multicolumn{4}{|l|}{ Employment } \\
\hline Employed & 40.2 & 40.3 & 40.1 \\
\hline Unemployed & 55.0 & 54.6 & 55.4 \\
\hline Student/retired/disabled & 4.8 & 5.1 & 4.5 \\
\hline \multicolumn{4}{|l|}{ Current abuse or dependence diagnosis } \\
\hline Cocaine & 70.5 & 72.7 & 68.2 \\
\hline Stimulants & 7.7 & 8.5 & 6.8 \\
\hline Opiates & 25.6 & 25.6 & 25.6 \\
\hline Marijuana & 27.2 & 27.8 & 26.6 \\
\hline Alcohol & 56.1 & 59.7 & 52.5 \\
\hline PTSD diagnosis ( $\%$ meeting full criteria) & 80.4 & 76.7 & 84.2 \\
\hline \multicolumn{4}{|l|}{ Lifetime traumatic experiences } \\
\hline Child physical abuse & 58.7 & 61.1 & 56.3 \\
\hline Adult physical abuse & 84.8 & 83.4 & 86.2 \\
\hline Child sexual abuse & 70.1 & 73.6 & 66.7 \\
\hline Adult sexual abuse & 67.6 & 65.1 & 70.1 \\
\hline Transportation accident & 72.7 & 72.2 & 73.3 \\
\hline Life-threatening illness & 39.8 & 41.5 & 38.1 \\
\hline Exposed to violent death & 19.3 & 16.5 & 22.2 \\
\hline
\end{tabular}

Note. Values are means (with standard deviations in parentheses) unless otherwise noted. PTSD $=$ posttraumatic stress disorder.

${ }^{a}$ There were no statistical differences between treatment groups on any variable. 


\section{Table 2}

Global Fit

\begin{tabular}{ccc}
\hline & \multicolumn{2}{c}{ BIC/entropy } \\
\cline { 2 - 3 } No. of classes & Alcohol & Cocaine \\
\hline 2 & $4,814 / .820$ & $4,605 / .822$ \\
3 & $4,710 / .785$ & $4,501 / .786$ \\
4 & $4,753 / .746$ & $4,544 / .754$ \\
\hline
\end{tabular}

Note. $\mathrm{BIC}=$ Bayesian information criterion. 
